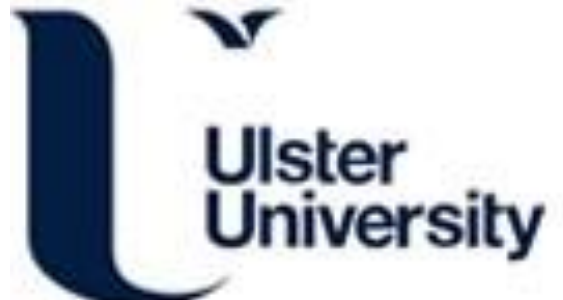

\section{A Substance Use Treatment Programme for Mentally III Forensic Patients in an Australian Setting: A Pilot Study of Feasibility, Acceptability and Preliminary Efficacy}

Mc Fadden, D., Barrett , E., \& Prior , K. (2020). A Substance Use Treatment Programme for Mentally III Forensic Patients in an Australian Setting: A Pilot Study of Feasibility, Acceptability and Preliminary Efficacy. International Journal of Mental Health and Addiction. https://doi.org/10.1007/s11469-020-00348-3

Link to publication record in Ulster University Research Portal

Published in:

International Journal of Mental Health and Addiction

Publication Status:

Published (in print/issue): 12/07/2020

DOI:

10.1007/s11469-020-00348-3

Document Version

Author Accepted version

\section{General rights}

Copyright for the publications made accessible via Ulster University's Research Portal is retained by the author(s) and / or other copyright owners and it is a condition of accessing these publications that users recognise and abide by the legal requirements associated with these rights.

\section{Take down policy}

The Research Portal is Ulster University's institutional repository that provides access to Ulster's research outputs. Every effort has been made to ensure that content in the Research Portal does not infringe any person's rights, or applicable UK laws. If you discover content in the Research Portal that you believe breaches copyright or violates any law, please contact pure-support@ulster.ac.uk. 
A substance use treatment programme for mentally ill forensic patients in an Australian setting: A pilot study of feasibility, acceptability and preliminary efficacy Daniel McFadden ${ }^{1,2}$, Katrina Prior ${ }^{1}$ and Emma L. Barrett ${ }^{1}$

${ }^{1}$ Matilda Centre for Research in Mental Health and Substance Use, The University of Sydney, Sydney, New South Wales, Australia

${ }^{2}$ Justice Health and Forensic Mental Health Network, Sydney, Australia

Corresponding Author: Mr Daniel McFadden

Postal Address: The Matilda Centre, Level 6, Jane Foss Russell building (G02),

The University of Sydney, NSW 2006, Australia

Email:dmcf4572@uni.sydney.edu.au 


\begin{abstract}
This study aims to evaluate the feasibility, acceptability and preliminary efficacy of an Australian-adapted Substance Use Treatment Programme (SUTP) among mentally ill offenders in an Australian secure forensic setting. A single-group non-controlled pilot trial was conducted. Four SUTP treatment groups were run simultaneously $(n=39)$ with sessions held weekly over 12 weeks. Feasibility was assessed by examining attrition, study retention and motivation for treatment, and measures of therapeutic alliance, client satisfaction and qualitative interviews were used to determine acceptability. Preliminary efficacy was assessed using self-report questionnaires implemented at baseline and post-intervention measuring drug related locus of control, confidence to resist substances in the future and beliefs about substance use. Low attrition, high levels of satisfaction and moderately positive levels of therapeutic alliance were found. Participants also demonstrated a significant shift towards an internal drug related locus of control upon completion of SUTP. SUTP is a promising intervention for mentally ill offenders with a history of substance use in Australian forensic services. A randomised controlled trial is warranted to rigorously evaluate the efficacy of SUTP in this setting.
\end{abstract}

Keywords: Forensic, mental illness, offenders, substance use, treatment, group interventions 


\section{Introduction}

Mental disorders occur at high rates among prisoners worldwide (Fazel, 2012). In Australia, rates of mental disorders among these individuals are disproportionately high when compared with the general population. The 12-month prevalence of any mental disorder in prisoners is $46 \%$, far exceeding that of a matched sample of the Australian general population $(16 \%)$, indicating that prisoners are nearly five times more likely to have a mental disorder when compared with the community $(\mathrm{OR}=4.7,95 \% \mathrm{CI}=4.0-5.6$; Butler, Andrews, Allnutt, Sakashita, Smith, \& Basson, 2006). A common point at which Australian prisoners are identified as having a mental disorder is during the course of a special hearing or trial where an individual can be found 'Not Guilty by Reason of Mental Illness' by a judge or jury. This is determined when the person is found not to have the requisite responsibility to be found guilty of the crime because of their mental illness (Boyd-Caine \& Chappell, 2005). In New South Wales (NSW), the most populous state in Australia, 364 individuals were found not guilty by reason of mental illness between 1990 and 2010 (Hayes, Kemp, Large, \& Nielssen, 2014). A more recent survey of forensic inpatients in NSW reported that the most common diagnosis among people who are found not guilty by reason of mental illness is a psychotic disorder, with a prevalence of $83 \%$ (Justice Health and Forensic Mental Health Network; JHFMHN, 2016). Other mental disorders in this group included personality disorders (13\%), depression (13\%), and neurocognitive disorders (11\%). The study reported that the most common unlawful act committed by NSW forensic patients was murder (41\%), followed by assault resulting in serious injury (16\%), aggravated sexual assault (15\%), aggravated robbery $(8 \%)$ and attempted murder $(6 \%$; JHFMHN, 2016). These patients are placed under the supervision of the Mental Health Review Tribunal and are treated in a secure hospital setting by NSW Health until they are deemed well enough to be granted conditional release into the community. 
Rates of substance use disorders in prisoners are also extremely high (Fazel, Bains, \& Doll, 2006), with between half to two-thirds (55\%-66\%) of Australian adult prisoners diagnosed with a substance use disorder in the past 12 months; a rate that is 11 times higher than adults in the general population (Butler et al., 2006; Butler, Indig, Allnutt, \& Mamoon, 2011). Furthermore, empirical evidence among both national and international samples has demonstrated that mentally ill offenders, in particular, experience high rates of co-occurring substance use disorders (Butler et al., 2011; Ogloff, Lemphers, \& Dwyer, 2004; Scott, Whyte, Burnett, Hawley, \& Maden, 2004; Tyler, Miles, Karadag, \& Rogers, 2019; Wright, Walters, \& Strang, 2016). A 2018 study of mentally ill patients the NSW secure forensic hospital reported that three-quarters of patients (75\%) had a diagnosed substance use disorder (Eagle, Ma, \& Sinclair, 2019). This of significant importance, given that individuals with cooccurring mental illness and substance use disorders present with a much more complex clinical profile and an increased risk for reoffending (Barrett, Teesson, \& Mills, 2014; Baxter, Rabe-Hesketh, \& Parrott, 1999; De Burca, Miles, \& Vasquez, 2013), with previous research demonstrating an association between the use of alcohol and drugs and the reconviction of released forensic patients (Scott et al., 2004; Smith \& Trimboli, 2010). There is a critical need to identify and address co-occurring substance use disorders in mentally ill offenders, not only to improve clinical outcomes for this population, but to significantly reduce risk of reoffending in the community (Pickard \& Fazel, 2013).

Despite the high prevalence and harms associated with substance use disorders among mentally ill offenders (Clarke, Davies, Hollin, \& Duggan, 2011; Johnson, Smith, Crowe, \& Donovan, 1993), there continues to be a paucity of research examining effective treatment approaches for this population. Psychoeducation tends to be the predominant model of care for mentally ill offenders with a substance misuse history, despite previous research showing that this treatment approach does not always inspire cognitive or behavioural change 
(Sandbrook, Clark, \& Cocksedge, 2015). In contrast, research strongly supports the use of cognitive behavioural therapy (CBT) as a treatment for substance use disorders in both individual and group settings (Jhanjee, 2014; McHugh, Hearon, \& Otto, 2010). Moreover, international guidelines recommend the use of CBT in the treatment of substance use disorders (National Institute of Health and Care Excellence, 2007) and a number of metaanalyses report the positive effects of CBT on the recidivism of offenders (Landenberger \& Lipsey, 2005; Lipsey, Chapman, \& Landenberger, 2001; Mpofu, Athanasou, Rafe, \& Belshaw, 2018).

There is a critical need for further evaluation of CBT-based approaches for addressing substance use disorders in mentally ill offenders in Australia. Emerging research conducted in medium secure settings in the United Kingdom (UK) has demonstrated promising results. One study found that 16 weekly group sessions of a CBT-based programme for substance use $(n=37)$ improved external motivation for treatment compared to those who completed a psychoeducation-based intervention $(n=43)$, however, both groups experienced high drop-out rates of $46 \%$ and $29 \%$, respectively (Tibber, Piek, \& Boulter, 2015). Another CBT-based programme comprised of 16 weekly group sessions plus 5 individual sessions delivered to 23 patients in a medium secure unit demonstrated a statistically significant pre to postintervention increase in inpatient's confidence to resist their drug of choice (Oddie \& Davies, 2009). This program also proved to be highly acceptable to participants, as evidenced by high attendance and positive feedback in qualitative interviews at post-intervention (Oddie \& Davies, 2009). A similar 10 session group programme was implemented in East London, with no formal evaluation completed (Wood, Patel, Skinner, \& Thomson, 2009). However, qualitative feedback from key professional staff involved in service provision indicated that it was useful in building therapeutic relationships with clients and in helping them to 
understand the impact that substance use can have on individuals, which has clear benefits for themselves and their clients (Wood et al., 2009).

High secure forensic settings have also demonstrated promising findings for the efficacy of using a CBT-based treatment approaches for substance use disorders in the UK. An evaluation of a weekly substance use group treatment programme delivered to 30 male patients in Wales over a 9- to 14-month period found a significant pre-post assessment change in patient reported ambivalence, which was interpreted as an acknowledgement of the problematic nature of substance misuse (Morris \& Moore, 2009). Another high secure study in Scotland involved an evaluation of a coping and social skills relapse prevention group programme ( 28 three-hour sessions over a 14-week period) delivered to 83 male participants (Ritchie, Weldon, Freeman, MacPherson, \& Davies, 2011). Those who completed the programme showed a significant post-intervention increase in confidence to resist their primary substance of choice in the future. Although the programme focused primarily on social skills, it demonstrated the beneficial changes that dual diagnosis targeted interventions can achieve.

One of the most promising CBT-based programmes for forensic patients is the Substance Use Treatment Programme (SUTP; Miles, 2015). An earlier version of this programme containing 24 one-hour, group sessions delivered weekly in a medium secure forensic setting was evaluated with 19 participants in the UK (Miles, Dutheil, Welsby, \& Haider, 2007). In this study, SUTP was found to significantly increase abstinence rates, with $74 \%(n=14)$ remaining abstinent at the end of the programme. The authors acknowledge that the high rates of abstinence could be due to both legal restrictions and incentives, such as day leave, contributing to increased motivation (Miles et al., 2007). Derry and Batson (2008) also conducted a preliminary analysis into the effectiveness of this 24 -session version of SUTP in a medium security forensic mental health service $(n=61)$. They found that those who 
completed SUTP (defined as attending at least 18 out of 24 weekly sessions) were significantly less likely to use drugs or alcohol after discharge (50\% had used drugs), compared to those who did not complete it ( $74 \%$ had used drugs). Additionally, the SUTP participants spent a significantly greater proportion of time in the community following discharge (89\%), compared to those who didn't receive the treatment $(77 \%)$. The SUTP was refined and modified to consist of 12 two-hour, group sessions delivered weekly to medium secure forensic patents (Miles, 2015). Due to the restrictive environment, the study did not focus on abstinence rates but rather on measured cognitive change such as participants adaptive beliefs around substance use and cravings. Significant pre- to post-treatment improvements were found, as well as high satisfaction levels with the programme (Miles, 2015).

These promising findings of the SUTP make it a relevant programme to address the gaps in practice in Australian forensic secure services. There is a critical need for evidencebased treatment approaches for offenders with mental health and drug and alcohol comorbidity. An evaluation of SUTP outside of the UK will determine its generalisability to participants in Australia. Additionally, the SUTP has been tested predominantly in medium secure settings, leaving a need for further evaluation in a high secure setting. As such, this study aims to evaluate the feasibility, acceptability and preliminary efficacy of the SUTP among forensic inpatients in an Australian high secure forensic hospital. This is the first study to evaluate the CBT-based SUTP among mentally ill offenders in Australia, which will directly inform clinical practice and contribute significantly to global knowledge in this area.

\section{Method}

\section{Design and setting}

The current study involves a single-group non-controlled pilot trial, conducted in a high secure forensic mental health facility in NSW. The hospital admits male and female 
mentally ill patients aged 18 years and over who have been in contact with the criminal justice system, as well as high-risk civil patients. Ethical approval for this study was granted by the JHFMHN Human Research Ethics Committee (2019/ETH00244) and the Aboriginal Health and Medical Research Council of NSW Human Research Ethics Committee (AHMRC: 1533/19). Site Specific approval was also granted by the JHFMHN (2019/STE00375). The pilot study was conducted from September 2019 to December 2019, during which time participants were recruited, received the SUTP and completed pre- and post-intervention assessments.

\section{Participants and procedure}

All participants $(N=39)$ were inpatients of the forensic hospital who had been identified by their multi-disciplinary treating teams as requiring a substance misuse intervention and subsequently referred to the SUTP. Patients were informed of the pilot study by their treating team, after which they were given the opportunity to participate. Informed written consent was obtained from all interested participants by a researcher-clinician who was independent of the clinical care of the patient. Reassurance was provided that a patient's decision whether or not to participate in the research study would not impact the care or treatment provided at the hospital. An Aboriginal mental health professional was present during the attainment of informed consent from all patients who identified as being of Aboriginal and Torres Strait Islander descent to ensure that historical factors did not influence the participants consent to partake in the research. Patients who did not wish to take part in the study, or who were not well enough to provide consent (as assessed by their treating team), were still offered to take part in the programme as part of their routine clinical care provision. However, in such instances, they were not included in the current research study. 
All referred patients who were interested in being involved in the research were assessed for eligibility using a screening tool designed for the purposes of this study. Participants were included if they were aged over 18 years and experienced harmful or hazardous alcohol use (as assessed by the Alcohol Use Disorders Identification Test [AUDIT] >=8; Babor, Higgins-Biddle. Saunders \& Monteiro, 2001) or drug use (as determined by the Drug Use Disorders Identification Test [DUDIT] $>=6$ for males, $>=2$ for females; Berman, 2003). The AUDIT and DUDIT items were asked in relation to the year prior to incarceration. Participants were excluded if they had a severe cognitive impairment or if they were too unwell to receive the programme (as determined by their treating team), had difficulty speaking or comprehending English, or if they were unable to provide informed consent. Of the 52 patients who were referred for inclusion in the pilot SUTP, 39 were deemed eligible to be included and provided consent. Of those who were excluded, 10 patients declined to participate (nine refused the treatment and one declined to participate in the study), two were too unwell at the time of the treatment being offered, and one had been discharged prior to group commencement (see Figure I).

\section{[INSERT FIGURE I ABOUT HERE]}

Eligible participants were allocated to one of four concurrent SUTP groups (described below) and completed face-to-face assessments at baseline (i.e., pre-intervention) and 3months post-baseline (i.e., post-intervention). Baseline and post-intervention follow up assessments were administered by a researcher-clinician independent of the treating team and took approximately 45 minutes and 25 minutes to complete, respectively. Thirty-three participants $(84.6 \%)$ participated in the post-intervention assessments and qualitative interviews. Participants who dropped out during the first session $(n=3)$, were discharged from the hospital $(n=2)$, or refused to complete the follow-up assessment $(n=1)$ were considered lost to follow-up as they did not complete the post-intervention assessment. Thirty-one 
participants completed all acceptability and efficacy measures and three participants completed the acceptability measures only (total sample size for the CSQ-8 at postintervention was $n=33$ and the WAI was $n=32$ ).

\section{Enrolment}

\section{Allocation}

\section{Follow-Up}

Analysis

Figure I: Study flow diagram for the SUTP 


\section{The Substance Use Treatment Programme (SUTP)}

The SUTP comprises two stages containing six sessions in each stage. Table I outlines the contents of each of the stages and sessions. The programme was developed within a CBT framework and is underpinned by motivational interviewing (Miles, 2015). The SUTP typically contains a "New Horizon" group which are participant lead booster sessions, however, for the purposes of this pilot study, booster sessions were not offered. Participants were assigned to one of four concurrent groups of SUTP, each of which ran weekly sessions for 12 weeks and were facilitated by two Forensic Hospital Allied Health Professionals, per group. There was a maximum of 10 patients in each SUTP group at one time. Staff from the Forensic Hospital Allied Health team were trained in the delivery of the treatment programme by developer Dr Helen Miles via a two-day, face-to-face workshop.

[INSERT TABLE I ABOUT HERE] 
Table I.

Substance use treatment programme (SUTP) session plan outline

Stage 1

Week 1 Group introductions and building engagement - Opening discussions, using socratic questioning and substance use (e.g. role in society, participants lifestyle prior to hospital etc.)

Week 2 Building motivation for change - Motivational interviewing exercises (e.g. evaluation of the pros/cons of substance use, using a decisional balance process), identification of the negative effects of substance on different areas of life, goal setting to improve identified problem areas and formulation of personal motivational statements for abstinence

Week 3 Alcohol - Psychoeducation on the effects of alcohol, harm minimisation (e.g. alcohol units and safe limits quiz), role of alcohol on medication. Visual "pig liver" demonstration, to demonstrate the impacts of alcohol on the liver.

Week 4 Cannabis - Psychoeducation on the effects of cannabis particularly on mental health and relapse, links to personal experiences and multi-media props (video/newspaper)

Week 5 Other substances - Psychoeducation on the effects of other illicit substances (e.g. stimulants, hallucinogens, solvents and opiates), linking these substances to mental illness and offending behaviour

Week $6 \quad$ Substance use and offending behaviour - Examination of direct and indirect links between substance use and crimes/own offending behaviours (e.g. timeline reviews).

Stage 2

Week 7 Self-esteem - Creative activity to explore participants view of self without substances and increases their self-esteem

Week 8 Functional analysis of substance use - CBT exercises (e.g. ABC charts exploring past substance use) to explore the function/reason for past use, identification of future highrisk situations/triggers for use, alternative coping strategies/activities and problem solve any barriers to change.

Week 9 Assertiveness - Identification of different communication styles and improvement of assertive refusal skills through role play exercises, identification of appropriate social support networks

Week 10 Craving - Identification of craving and "urge surfing" strategies (e.g. cue cards), and management of negative feelings (e.g. relaxation exercises)

Week 11 "Set ups" and lapses - Identification of risks for relapse and exploration of recommitting to abstinence after a lapse (e.g. challenging negative thinking, roleplays)

Week 12 Relapse prevention plan - Review the past 11 weeks and development of an individual relapse prevention plan. 


\section{Adaptation of SUTP for the Australian setting}

Seven experts were consulted to adapt the content of the SUTP manual for forensic patients in the Australian setting. Experts included individuals with a lived experience of mental illness, current peer workers and past patients of the hospital, as well as staff who work closely with family and carers who represented their views on treatment programmes. Additionally, an Aboriginal health worker and Aboriginal peer worker consulted on the manual to advise on any cultural considerations or changes that would be needed. Based on the feedback obtained, amendments were made to the content, in particular the vignettes and language. All other aspects of the programme remained the same including the length, number and structure of sessions as well as the key therapeutic techniques.

\section{Measures}

Measures used to assess feasibility, acceptability and preliminary efficacy are described in detail in Table II and summarised below.

\section{[INSERT TABLE II ABOUT HERE]}

\section{Feasibility}

Treatment adherence was measured by attendance at each of the 12 SUTP sessions and study retention was assessed according to the follow-up rate at post-intervention. To assess motivation and reasons for entering treatment, the 9-item Treatment Entry Questionnaire (TEQ; Urbanoski \& Wild, 2012) was administered at baseline. As described in Table II, the TEQ includes three subscales regarding the motivation for entering treatment, including 'identified', 'introjected' and 'external' motivation. Total scores for each subscale range from 3 to 21, with higher scores on each subscale indicating higher levels of identified, introjected, and external motivation of motivation for entering treatment. 


\section{Acceptability}

Participant satisfaction with the SUTP was measured at post-intervention using the 8item self-report Client Satisfaction Questionnaire (CSQ-8; Attkisson \& Greenfield, 1996). Total scores range from 8 to 32, with higher scores indicating higher levels of satisfaction with the programme. Participants were also asked six open-ended questions on their experience of the treatment programme, including what aspects they found most and least helpful, what elements they would change, what impact the program has had on their life, and whether they had experienced similar treatment programmes in the past.

Therapeutic alliance was measured using the 12-item Working Alliance InventoryShort Form (Horvath, 1991). Total scores range from 12 to 84 with higher scores being indicative of a more positive therapeutic alliance and improved client outcomes (Sturgiss, Rieger, Haesler, Ridd, Douglas, \& Galvin, 2019).

\section{Preliminary efficacy}

Participants were invited to take part in a face-to-face, structured baseline assessment that collected information on demographic characteristics (e.g., date of birth, gender, country of birth), drug use history, treatment history, confidence to resist substance use in the future, drug related locus of control, multi-dimensional locus of control and beliefs about substance use (the validated measures used to assess these constructs are discussed below and described in detail in Table II). At post-intervention, participants were invited to take part in an assessment that re-assessed confidence to resist substance use, drug related locus of control, multi-dimensional locus of control, and beliefs about substance use. It was not possible to measure substance use-related behavioural changes in the restricted environment of the 
forensic hospital. Participant attitudes and beliefs towards substance use were measured to provide insight into future substance related behaviours.

To assess level of confidence in being able to cope with high-risk situations with respect to their previous addictive behaviours, participants were administered the 8-item Drug Taking Confidence Questionnaire (DTCQ-8;Sklar \& Turner, 1999). Scores on the DTCQ range from 0 to 100 , whereby higher scores are indicative of greater confidence to resist urges to use substances.

Locus of control was measured by 15-item Drug Related Locus of Control (DR-LOC; Ersche, Turton, Croudace, \& Stochl, 2012) and 24-item Levenson Multi-Dimensional Locus of Control Questionnaire (MD-LOC; Levenson, 1973). DR-LOC assesses the extent to which a person believes that the outcome of 15 specific drug-related events are under their personal control (i.e., internal locus of control) or the influence of external circumstances (i.e., external locus of control). Scores range from 0 to 15 ; higher scores indicate a stronger belief of external locus of control. Levenson MD-LOC (Levenson, 1973) consists of three subscales addressing different beliefs about locus of control: internal (control over own life), powerful others (other's control over one's life) and chance (life is a matter of chance). A higher rating on internal locus of control subscale (MD-LOC Internal) indicates a strong internal locus of control, and high scores on the powerful others (MD-LOC Powerful Others) and chance (MD-LOC Chance) subscales indicates a strong external locus of control.

Beliefs about substance use, such as whether participants believe they need substances to cope or fulfil a need, was assessed using a 20-item Beliefs about Substance Use (BSU) questionnaire (Beck, Wright, Newman, \& Liese, 1993). Total scores range from 20 to 140, with higher scores indicating more maladaptive beliefs maladaptive beliefs. 
Table II. Summary of the validated scales used in the current study

\begin{tabular}{lll}
\hline Measure Description & Psychometric properties
\end{tabular}

\section{Feasibility}

Treatment Entry

Questionnaire

(TEQ; Urbanoski \&

Wild, 2012)

\section{Acceptability}

Client Satisfaction

Questionnaire

(CSQ-8; Attkisson

\& Greenfield,

1996)

Working Alliance Inventory-Short

Form (WAI-SF;

Horvath \&

Symonds, 1991)
The 8-item TEQ-9 includes three subscales that include the degree to which the person entered treatment because: i) the participant identifies personally with the positive value of behaviour change and are committed by personal choice to the goals of the treatment programme (i.e., 'identified' motivation), ii) the participant identifies with what will happen negatively if they do not change such as the impact of their own feelings of guilt on themselves (i.e., 'introjected' motivation), and iii) attendance is due to an external referral, identified pressure, ultimatum or another coercive social force (i.e. 'external' motivation). Four items assess each of the three subscales, rated on a 7-point Likert scale ranging from 1 'strongly disagree' to 7 'strongly agree' (total scores for each subscale range from 3 to 21). A weighted mean is calculated for each motivational subscale, with higher scores indicating higher levels of identified, introjected, and external motivation of motivation for entering treatment. A high score for one type of motivation does not prevent a high score for another.

The 8 items of the CSQ are scored on a Likert scale from 1 to 4 , with 1 indicating low satisfaction and 4 high satisfaction. Total scores range from 8 to 32, with higher scores indicating higher levels of satisfaction with the programme. Due to the scales single factor structure, the results were compared with external data that constitutes an appropriate norm (e.g., the multi-service setting means and standard deviation results presented in previous studies (Attkisson \& Greenfield, 1996; Attkisson \& Zwick, 1982)

The 12 items on the WAI-SF are rated on a 7-point Likert scale ranging from 1 'never' to 7 'always', with total scores ranging from 12 to 84 (items 4 and 10 are reverse coded). Higher scores are indicative of a more positive therapeutic alliance and improved client outcomes (Sturgiss et al., 2019)
The TEQ has high internal consistency in a substance misuse residential population (internal motivation $\alpha=.90$, introjected motivation $\alpha=.83$, and external motivation $\alpha=.87$; Urbanoski \& Wild, 2012). In the current study, the TEQ showed moderate reliability for internal motivation $(\alpha=.67)$ and introjected motivation $(\alpha=.69)$ and high reliability for external motivation $(\alpha=.84)$. While the internal and introjected subscale scores are just below the standard threshold of internal reliability (Cronbach's alpha $<0.7)$, it has been argued that a reliability of $>.60$ is acceptable for scales of less than ten items (Loewenthal, 2018).

The CSQ-8 demonstrates high levels of internal consistency in mental health settings (Cronbach's $\alpha=0.83-0.93$;

Attkisson \& Greenfield, 1996) and residential substance use settings (Cronbach's $\alpha=0.92$; Kelly, Kyngdon, Ingram, Deane, Baker, \& Osborne, 2018). The CSQ-8 was also shown to be a reliable measure in the current study, as determined by a Cronbach's alpha of $\alpha=0.87$

The WAI-SF demonstrates high levels of reliability in therapeutic settings (Cronbach's $\alpha=0.85$; Sturgiss et al., 2019) and rehabilitation settings (Cronbach's $\alpha=0.93$; Paap, Schrier, \& Dijkstra, 2019), and moderate to high levels of reliability in forensic mental health rehabilitation settings (Cronbach's $\alpha=0.94$; Donnelly, Lynch, Devlin, Naughten, 


\section{Preliminary efficacy}

Drug Taking

Confidence

Questionnaire

(DTCQ-8; Sklar \&

Turner, 1999)

Drug Related

Locus of Control

(DR-LOC; Ersche

et al., 2012)

Levenson Multi-

Dimensional Locus

of Control

Questionnaire

(MD-LOC)

Beliefs about

Substance Use

(BSU)

questionnaire (Beck

et al., 1993)
The 8-item DTCQ-8 identifies eight future high-risk situations: unpleasant emotions, physical discomfort, pleasant emotions, testing personal control, urges and temptation to use, conflict with others, social pressure to use, and pleasant times with others. The participants confidence to resist the urge to use alcohol or other drugs in these situations is measured on a 6-point scale from 0 'not at all confident' to 100 'very confident'. A mean score is computed (range $=0$ to 100 ), whereby higher scores are indicative of greater confidence to resist urges to use substances.

The 15-item DR-LOC assesses the extent to which a person believes that the outcome of an event is under their personal control (i.e., internal locus of control) or the influence of external circumstances (i.e., external locus of control). Individual items are binary scored as either 0 'internal' or 1 'external'. Items 1, 3, 5, 8, 11, 14 and 15 are reverse coded and then all items are summed. Scores range from 0 to 15 ; higher scores indicating a stronger belief of external locus of control.

Levenson MD-LOC (Levenson, 1973) is a 24-item self-report questionnaire consisting of three subscales of eight items each: internal (control over own life), powerful others (other's control over one's life) and chance (life is a matter of chance). Responses are scored on a 6-point Likert scale ranging from -3 'strongly disagree ' to +3 'strongly agree'. A higher rating on internal locus of control subscale (MD-LOC Internal) indicates a strong internal locus of control, and high scores on the powerful others (MD-LOC Powerful Others) and chance (MD-LOC Chance) subscales indicates a strong external locus of control. Participants receive three subscale scores and it is possible to score high or low on all three dimensions (Kourmousi, Xythali, \& Koutras, 2015).

The 20 items focus on self-efficacy, self-confidence to stop using substances, and self-blaming thoughts. Items are rated on a 7-point Likert scale, scored from 1 'strongly disagree' to 7 'strongly agree' (range $=20$ to 140), with higher scores indicating more maladaptive beliefs.
Gibbons, Mohan, \& Kennedy, 2011) including in the current study (Cronbach's $\alpha=0.78$ ).

The DTCQ-8 has proven high internal consistency in evaluating treatment outcomes among those with alcohol and other drug problems (Cronbach's $\alpha=0.98$; Sklar, Annis, \& Turner, 1997; Sklar \& Turner, 1999), and was also shown to be highly reliable in the current study among forensic inpatients (Cronbach's $\alpha=0.91$ ).

The DR-LOC has demonstrated high internal consistency among clients engaged with both residential and outpatient drug treatment services (Cronbach's $\alpha=0.81$; Hall, 2001), internal consistency in this study was moderate, Cronbach's $\alpha=0.66$.

The internal consistency of the measure has been shown in mental health hospital inpatients (Cronbach's $\alpha=0.67$ for MD-LOC Internal, 0.82 for MD-LOC Powerful Others and 0.79 for MD-LOC Chance; Levenson, 1973), as well as in the current study of forensic inpatients (Cronbach's $\alpha=.83$, $\alpha=.85$ and $\alpha=.67$ for the MD-LOC Internal, Powerful Others and Chance subscales, respectively). While the internality subscale score showed relatively low levels of internal consistency (Cronbach's alpha $<0.7$ ), the MD-LOC items sample a wide variety of different situations and as such this was to be expected (Levenson, 1973).

The BSU has been found to have good internal consistency for alcohol dependent patients (Cronbach's $\alpha=0.91$; Aslan, Türkçapar, Eser, \& Uğurlu, 2012), and mentally ill offenders in the current study (Cronbach's $\alpha=.92$ ). 


\section{File review}

To gain an understanding of the demographic and clinical characteristics of the participants, informed consent was obtained from almost all participants $(97.4 \% ; n=39)$ to collect existing data from participant medical files stored at the hospital. This included sociodemographic and clinical information such as date of birth, gender, participants, legal status, unlawful act, history of substance use, history of attending psychoeducational substance use treatment programmes in the forensic hospital, treatment history and diagnostic information on all serious mental disorders including schizophrenia, paranoid schizophrenia, schizoaffective disorder, substance use disorders, personality disorders and mood disorders.

\section{Statistical analysis}

Descriptive statistics were reported for outcomes relating to sociodemographic characteristics, feasibility, acceptability and preliminary efficacy. Inferential statistics were carried out to examine pre- and post-intervention comparisons. Data analyses were conducted using SPSS version 24, with significance levels set at $p<.05$ (95\% confidence intervals [CI]). Both parametric (T-tests) and non-parametric tests (Wilcoxon Signed Ranks Test) were carried out, dependent upon the distribution of the data. Effect sizes were calculated and are reported as Cohen's $d$ for normally distributed data, with values indicating a small $(d=0.2)$, medium $(d=0.5)$ or large $(d=0.8)$ effect sizes (Cohen, 2013), or $r$ for skewed data, with values indicating a small $(r=0.1)$, medium $(r=0.3)$ or large $(r=0.5)$ effect sizes (Fritz, Morris, \& Richler, 2012). A thematic analysis of the qualitative data on treatment acceptability was performed using NVivo version 12 data analysis software. Data was coded and collated under specific themes and reported upon based on these themes. 


\section{Results}

\section{Sample characteristics}

Table III displays the sociodemographic and substance use characteristics of the sample at baseline $(n=39)$. Thirty-two participants were male $(82.1 \%)$ and seven were female $(17.9 \%)$.

[INSERT TABLE III ABOUT HERE] 
Sociodemographic, mental health, substance use and substance use treatment characteristics of the sample $(n=39)$

\begin{tabular}{|c|c|}
\hline Characteristic & Statistic \\
\hline Male gender, $\%(n)$ & $82.1(32)$ \\
\hline Age, $M(\mathrm{SD})$ & $41.7(11.09)$ \\
\hline Australian born, $\%(n)$ & $87.2(34)$ \\
\hline Aboriginal and Torres Strait Islander, \% $(n)$ & $12.8(5)$ \\
\hline \multicolumn{2}{|l|}{ Primary mental health diagnosis } \\
\hline Schizophrenia, $\%(n)$ & $69.2(27)$ \\
\hline Schizoaffective disorder, \% $(n)$ & $20.5(8)$ \\
\hline Other $^{1}, \%(n)$ & $10.3(4)$ \\
\hline \multicolumn{2}{|l|}{ Legal Status } \\
\hline Forensic (NGMI), \% $(n)$ & $92.3(36)$ \\
\hline Limiting term, $\%(n)$ & $5.1(2)$ \\
\hline Unfit to plead, $\%(n)$ & $2.6(1)$ \\
\hline \multicolumn{2}{|l|}{ Unlawful Act resulting in admission to Forensic Hospital } \\
\hline Murder, $\%(n)$ & $43.6(17)$ \\
\hline Grievous bodily harm, \% (n) & $25.6(10)$ \\
\hline Assault, \% $(n)$ & $10.3(4)$ \\
\hline Robbery, \% $(n)$ & $7.7(3)$ \\
\hline Arson, $\%(n)$ & $5.1(2)$ \\
\hline Sexual offences, $\%(n)$ & $5.1(2)$ \\
\hline Other $^{2}, \%(n)$ & $20.6(10)$ \\
\hline Age of first alcohol/drug use, $M$ (SD) & $14.0(3.2)$ \\
\hline \multicolumn{2}{|l|}{ First drug used $(n=37)$} \\
\hline Alcohol, \% $(n)$ & $86.5(32)$ \\
\hline Cannabis, \% $(n)$ & $5.4(2)$ \\
\hline Benzodiazepines, \% $(n)$ & $2.7(1)$ \\
\hline Meth/amphetamines, \% $(n)$ & $2.7(1)$ \\
\hline Heroin, $\%(n)$ & $2.7(1)$ \\
\hline \multicolumn{2}{|l|}{ Most recent use of alcohol or drug $(n=38)$} \\
\hline In current forensic hospital placement, \% $(n)$ & $7.9(3)$ \\
\hline In custodial placement, $\%(n)$ & $18.4(7)$ \\
\hline In the community, $\%(n)$ & $73.7(28)$ \\
\hline \multicolumn{2}{|l|}{ Type of substance most recently used ( $n=38$ ) } \\
\hline Alcohol, \% $(n)$ & $47.4(18)$ \\
\hline Cannabis, \% $(n)$ & $36.8(14)$ \\
\hline Ecstasy, $\%(n)$ & $7.9(3)$ \\
\hline Meth/amphetamines, \% $(n)$ & $36.8(14)$ \\
\hline Heroin, $\%(n)$ & $5.3(2)$ \\
\hline Other opiates, $\%(n)$ & $2.6(1)$ \\
\hline \multicolumn{2}{|l|}{ Main current drug of concern $(n=38)$} \\
\hline Alcohol, \% $(n)$ & $39.5(15)$ \\
\hline Cannabis, $\%(n)$ & $26.3(10)$ \\
\hline Meth/amphetamines, \% $(n)$ & $26.3(10)$ \\
\hline Heroin, $\%(n)$ & $5.3(2)$ \\
\hline Other opiates, $\%(n)$ & $2.6(1)$ \\
\hline $\begin{array}{l}\text { Ever sought treatment from a health professional or service for alcohol } \\
\text { or other drug use }(n=37)\end{array}$ & $43.2(16)$ \\
\hline Age first sought treatment from a health professional or service ${ }^{3}, M(\mathrm{SD})$ & $22.8(5.6)$ \\
\hline First type of treatment sought ${ }^{3}$ & \\
\hline Inpatient detox, $\%(n)$ & $43.8(7)$ \\
\hline
\end{tabular}


Inpatient mental health unit, \% (n)

$12.5(2)$

Outpatient counselling, $\%(n)$

$6.3(1)$

Methadone/buprenorphine maintenance, $\%(n)$

$6.3(1)$

Community mental health team, \% (n)

$6.3(1)$

Current Forensic Hospital placement, \% (n)

$6.3(1)$

Custodial health services, $\%(n)$

$6.3(1)$

Community health programme, $\%(n)$

$6.3(1)$

Alcoholics/narcotics anonymous, \% (n)

Previously participated in the "psychoeducational addressing substance

${ }^{1}$ Bi-polar disorder or automatism,

${ }^{2}$ Attempted murder, dangerous driving occasioning death, soliciting to commit murder

${ }^{3}$ Of the 16 participants who had previously sought treatment for their alcohol or other drug use 


\section{Feasibility}

Participant adherence to the treatment programme was high, with $97.4 \%(n=38)$ attending at least one session and $61.5 \%(n=24)$ attending all 12 sessions. The median number of sessions attended was 12 (range $=0-12$ ). Reasons for non-attendance at sessions varied. The principal reason was patient refusal (62.0\%), followed by patients being discharged from hospital (24.1\%), appointment conflicts (8.6\%) and being unable to go outside to attend the group because of poor air quality due to the NSW bushfires and associated health conditions accounted (5.2\%).

The follow-up rate was also high, with $84.6 \%(n=33)$ participating in the postintervention assessments. In relation to treatment motivation, participants scored an average of $M=24.54(\mathrm{SD}=5.19)$ on the identified motivation subscale of the TEQ-9, $M=11.59$ $(\mathrm{SD}=5.34)$ on the introjected subscale and $M=11.10(\mathrm{SD}=5.90)$ on the external subscale. This indicates the participants were primarily motivated to enter treatment by the value of positive behaviour change and less motivated by the potential negative consequences of not changing or other external pressures.

\section{Acceptability}

Mean scores on the CSQ-8 at post-intervention were $M=28.6(\mathrm{SD}=3.57)$, indicating high levels of satisfaction with the programme. Two-thirds $(63.6 \%)$ of participants reported that they were generally "very satisfied" with the service they received, while the remaining third (33.3\%) reported themselves as being "mostly satisfied". Just over half $(54.5 \%)$ reported that the service "helped a great deal" with their problem, while $45.5 \%$ stated it "helped somewhat".

The mean score for the WAI-SF was $M=69.13$ (SD=7.43). One-third (33.3\%) reported that they "very often" felt confident in the facilitators ability to help them, with 
$48.5 \%$ reporting they "always" felt this. Over half (57.6\%) reported feeling that they and the facilitator "always" had mutually agreed upon goals with one-third (30.3\%) reporting that they "very often" had mutual goals. Table IV reports the qualitative data pertaining to positive and negative feedback on the programme, grouped into six specific themes relating to satisfaction with SUTP.

[INSERT TABLE IV ABOUT HERE] 
Table IV.

Qualitative findings relating to satisfaction with SUTP

Positive aspects of SUTP

Theme 1: Visual impact of group sessions

Theme 2: Craving, coping, triggers
"They didn't just sit there and tell us like 'bang, bang, bang, yeah, yeah, yeah', they actually showed us"

"It was quite good the realisation of how much money you actually spend where, you know, how when you look at that total, you think 'oh, you could've bought this, you could've bought this, you could've had this'. That was a bit of a wakeup call"

"Triggers, it dealt with triggers really well and everybody, most people in the group, were really honest about triggers, so yes, I like how it dealt with triggers"

"And the coping strategies so who you feel comfortable talking to and bringing it up with and stuff like that. Like a network of family, friends and social groups and all that stuff, and what to do if you are craving or how not to get yourself in that situation in the first place"

Theme 3: Feeling

"I didn't feel so alone in my journey"

supported, connected and understood

"I know that I'm not the only one like that"

Theme 4: Learning from others
"Learning from other people's mistakes in the group, the girls opening up about their addictions, and myself as well".

"In other groups you had to read and write a lot more, but this group I felt taught me more by listening and by talking"

Negative aspects of SUTP

Theme 1: Substance use discussion generates cravings

Theme 2: Irritation with other group participants
"Suppose concentrating on hearing other people's shares; if you like on their drug and alcohol use, sometimes it made you crave for it"

"Yeah, it made you think about it after group. You might think about it like 'aw, you know, gee, I wouldn't mind a drink' and someone was saying they 'ah yeah, wanted a drink', and you start thinking 'yeah I wouldn't mind a drink'”.

"All the things so people talk over other people and some of them they are very proud about their history. I don't like it"

"We set up the rules before the group start, about not talk over people. It's all on the board but people still do it" 


\section{Preliminary efficacy}

Table V outlines the baseline and post-intervention scores, $p$-values and effect sizes for each of the outcome measures. A paired-samples t-test was conducted to compare participant drug-taking confidence ratings, using the DTCQ-8. Mean scores on DTCQ-8 increased from baseline $(M=75.98)$ to post-intervention $(M=80.97)$, however, this difference was not statistically significant $(t(29)=-.923, p=.364)$. This indicates that the SUTP did not produce statistically significant improvements in participants' confidence to resist substances in future high-risk situations.

[INSERT TABLE ABOUT V HERE] 
Table V.

Baseline and post-intervention scores, $p$-values and effects sizes for each outcome variable

\begin{tabular}{lcccc}
\hline Measure & Baseline & Post -intervention & $p$-value & Effect size \\
\hline DTCQ-8, $M(S D ; n)$ & $75.98(23.29 ; 39)$ & $80.97(17.52 ; 31)$ & .364 & $d=0.24$ \\
DR-LOC, $M d n$ (Range; $n)$ & $1.13(1.00-1.53 ; 37)$ & $1.07(1.00-1.27 ; 31)$ & $<.001$ & $r=0.67$ \\
MD-LOC Internal, $M d n($ Range; $n)$ & $11.00(0.00-32.00 ; 38)$ & $40.00(17.00-48.00 ; 31)$ & $<.001$ & $r=0.86$ \\
MD-LOC Chance, $M(S D ; n)$ & $23.68(11.33 ; 38)$ & $24.07(10.41 ; 31)$ & .745 & $d=0.04$ \\
MD-LOC Powerful Others, $M(S D ; n)$ & $22.94(12.38 ; 35)$ & $24.19(11.74 ; 31)$ & .648 & $d=0.10$ \\
BSU, $M d n$ (Range; $n)$ & $42.00(20.00-108.00 ; 39)$ & $34.00(20.00-76.00 ; 31)$ & .684 & $r=0.07$ \\
\hline
\end{tabular}


A Wilcoxon Signed Ranks test revealed that participant scores on the DR-LOC reduced significantly from baseline $(M d n=1.13)$ to follow-up $(M d n=1.07 ; \mathrm{Z}=-3.66, p<.001)$, indicating a significant increase in levels of drug-related internal locus of control over this period. A Wilcoxon Signed Ranks test and two paired-samples t-tests were conducted to compare participant locus of control for the MDLOC subscales of Internal, Chance, and Powerful Others, respectively. Scores on the MD-LOC Internal subscale increased significantly from baseline $(M d n=11.00)$ to post-intervention $(M d n=40.00 ; \mathrm{Z}=-4.81, p<.001)$, indicating an increase in the level to which participants felt in control of their situation. No significant differences were detected in means scores between baseline and post-intervention for the MD-LOC Powerful Others $(t(29)=-3.28, p=.745)$ or the MD-LOC Chance $(t(29)=-$ $.461, p=.648)$ subscales.

A Wilcoxon Signed Ranks test showed that while participant beliefs about substance use on the BSU decreased from baseline $(M d n=42.00)$ to post-intervention $(M d n=34.00)$, these changes were not significant $(Z=-.407, p=.684)$, indicating that there were no significant improvements in beliefs around substance use following the intervention. 


\section{Discussion}

This study builds on previous work conducted in the UK evaluating the SUTP for mentally ill offenders and demonstrates the feasibility, acceptability and preliminary efficacy of this programme in the Australian forensic setting. The high levels of adherence to treatment sessions and retention in the study to post-intervention shows that the 12 -week group programme is feasible to run in a high secure forensic environment, which often has a number of competing daily priorities. The SUTP also demonstrated that it is an acceptable treatment program for this setting, as evidenced by its high satisfaction ratings and strong therapeutic alliance between facilitators and patients. Importantly, the programme demonstrated efficacy in increasing locus of control and beliefs. This is the first international adaptation of the SUTP and the first time a CBT-based substance use treatment programme has been trialed among mentally ill prisoners in a high secure setting in Australia. These positive findings indicate the programmes suitability for this population and the utility of adapting the program to the Australian high secure setting by consulting experts with a lived experience of mental illness and from the Australian indigenous community.

The sociodemographic characteristics of this sample were similar to those in previous reports of NSW forensic inpatients (JHFMHN, 2016), indicating the sample was generally representative of forensic inpatients in this setting. The number of Australian-born participants in this study $(87.2 \%)$ was slightly higher than that of the general population when last surveyed (71.5\%; Australian Bureau of Statistics, 2017). This could be attributed to lower levels of English among individuals born outside of Australia, excluding them from participating in SUTP. The Aboriginal and Torres Strait Islander representation in the sample was slightly below the average reported in NSW forensic mental health inpatient settings (12.8\% vs. $16.8 \%$; JHFMHN, 2016), which is possibly due to the increased prevalence of cognitive impairment among Aboriginal and Torres Strait Islander peoples who are 
incarcerated (Shepherd, 2017), potentially limiting their eligibility for referral. Prevalence of mental health diagnoses of the sample was in line with recent reports (Miles, 2015), with $69.2 \%$ of the sample having a primary diagnosis of schizophrenia. Almost half of participants (43.6\%) reported murder as the unlawful act resulting in admission to the forensic hospital. Participants in the programme had a history of substance use, however, the vast majority had not used drugs or alcohol since their placement in the forensic hospital (92.1\%). Almost half of participants $(45.9 \%)$ had sought treatment from a health professional for substance use issues and approximately two-thirds (61.5\%) had been referred to (and attended) the 'psychoeducational addressing substance use' group in the hospital previously.

\section{Feasibility}

The forensic mental health population are notoriously difficult to engage in substance related treatment programmes (McMurran, 2002; Miles, 2015). However, the high levels of adherence (i.e., attendance at sessions) reported in the current study demonstrate the feasibility of the programme for this group. Understandably, some treatment attrition occurred, which is typical of this environment due to some patient non-engagement in treatment programmes, particularly among patients who have been recently admitted and are at an earlier stage of the rehabilitation process. While attrition has been reported as a significant challenge in the treatment of female mentally ill offenders specifically (Long, Fulton, Fitzgerald, \& Hollin, 2010), no attrition occurred in the female sample in the current pilot study. This is a positive indicator of feasibility, when considering its future implementation among female patients in the current treatment service.

\section{Acceptability}

There were high levels of satisfaction reported by the participants on the CSQ-8, and the majority (96.9\%) reported feeling "very" or "mostly" satisfied with SUTP as a treatment programme. When compared with CSQ-8 satisfaction ratings of similar treatment 
programmes in custodial settings (Barrett, Indig, Sunjic, Sannibale, Sindicich, Rosenfeld, Najavits, \& Mills, 2015), the SUTP demonstrated high levels of satisfaction. The quantitative findings of high acceptability are reinforced by the positive qualitative data collected in the semi-structured interviews. For example, one participant reported "I felt I was included and listened to and the peers were really good supportive and helpful. It was a good group".

These findings are important as high levels of client satisfaction are often positively associated with improved treatment outcomes for patients in substance use programmes (Boden \& Moos, 2009; Carlson \& Gabriel, 2001). Moreover, treatment programmes have a better chance of longer-term successful implementation when patient groups record high levels of satisfaction (Andersson, Otterholt, \& Gråwe, 2017). The therapeutic alliance ratings were moderate when compared with similar substance use rehabilitation treatment programmes for offenders (Dekker, 2010). Moderate scoring in therapeutic alliance in an involuntary secure setting is interpreted as positive, especially given the challenges posed in overcoming power imbalances in these environments. Therapeutic alliance in the current study could have been impacted by the difficulty of operating a 12-week programme, where programme facilitators are occasionally absent due to sickness and other role priorities such as mental health review tribunals. Staff were often in the position where they substituted for each other, which naturally inhibits a strong patient facilitator relationship from growing. This could potentially be managed better if the programme is fully integrated into the service in the future, as groups could be postponed until the following week when the facilitators were available. Unfortunately, due to the restricted timeline and manualised research protocol for the current pilot trial, there was no opportunity to do this, and staff had to exercise a substitution system to ensure each group ran, irrespective of facilitator availability. 


\section{Preliminary Efficacy}

In relation to efficacy of the SUTP program, there were statistically significant improvements in drug related locus of control and internal locus of control. Participants felt more in control of their drug use and took on a much larger sense of responsibility surrounding it, following their participation in the treatment programme. This is a positive finding, and if sustained over the course of their time in the forensic hospital, would place them in a better position to rehabilitate through the hospital and maintain sobriety in the community. It should be noted that the internal consistency of this measure was $\alpha=0.66$ in this study, which indicates the outcome should be interpreted with a cautious optimism. In many forensic settings around the world, patients move through levels of security based upon risk assessments completed by their multi-disciplinary treating teams. Drug and alcohol use is specifically considered within these assessments, so patients who demonstrate increased insight and confidence in relation to their ability to control their substance using behaviours are likely to be assessed as lower risk. This could mean transitioning through the hospital at a quicker pace, dependent on other factors and potentially being cared for in less restrictive environments, such as low and medium secure settings. Due to their environment patients don't have physical temptations to use alcohol or drugs, when transferred to a less secure unit or into the community the availability of illicit substances increases (Dolan \& Kirwan, 2001). A stronger-drug related internal locus of control may place them in a better position to prevent relapse and ultimately readmission to forensic secure services. This would ultimately have positive consequences for society, with lower recidivism rates due to less substancerelated criminal activity.

Given the restrictive environment of forensic hospitals, it is not possible to measure substance use-related behavioural changes. Rather, in these settings, it is considered important to measure changes in participants attitudes and beliefs towards drugs or alcohol, 
which is indicative of their future behaviours. No significant differences were found in participants beliefs about substance use or their confidence to resist substance use in the future. Miles (2015) demonstrated significant improvements in beliefs about substance use from baseline to 6-month follow-up, which was possibly due to the inclusion of booster sessions that may have significantly contributed to the change in beliefs. Unlike Miles (2015), booster sessions were not offered in this pilot study. Participants demonstrated high levels of confidence in their ability to refuse substances at baseline, so the small size of confidence improvement could be due to their forced abstinence environment which can provide a 'false' confidence due to not being exposed to temptation to use in many years.

This study has a number of strengths including the recruitment of a suitable pilot sample size, high post-intervention follow-up rates and the completion of assessments by an individual independent of the treating team. Despite these strengths, there are a number of limitations in this study. Firstly, while the sample size was suitable for the purposes of the current study and is consistent with other studies in forensic hospitals worldwide (Miles, 2015; Morris \& Moore, 2009; Ritchie et al., 2011) it is too small to form any strong conclusions regarding efficacy. It may also be the reason some significant results weren't detected. There is also no control group to compare participant outcomes to, and as such, a randomised controlled trial of the SUTP with a larger sample of mentally ill offenders is required in order to rigorously examine efficacy of this intervention. The effect sizes reported here are intended to guide such future investigations. Although it is known which participants had previously attended the psychoeducational substance use group, no data was collected on other groups attended prior to commencement of the SUTP, including those that are not aimed at substance use but which use CBT to build insight into their mental illness and offending behaviour. It is possible that some patients would have gained skills in these preexisting groups, which they then transferred to their substance use behaviours. This could 
have contributed to the positive findings of this study. Future studies would benefit from controlling for previous or other concurrent treatments in their analysis. Finally, no longerterm follow up was completed with the participant group to determine if treatment effects would remain over a longer period of time. This is important in the context of SUTP as previous work in this area showed significant changes in beliefs about substance use at posttreatment (Beck et al., 1993) which were later found to be not significant at 6-month follow up (Miles, 2015). In conducting any longitudinal study, consideration of the impact of patient transfer to medium- and low-secure services, as well as to the community, will have to be made. Intensive and rigorous follow-up in these settings would be required.

Collectively, this study contributes further evidence that SUTP is an efficacious programme that is deemed as acceptable by patients and is highly feasible in forensic secure services. Given this is the first study outside the UK and the findings are positive, it provides optimism that SUTP could be implemented in forensic services across Australia. This will address substance use among mentally ill offenders, a common and harmful comorbidity which contributes to poorer clinical outcomes and increased risk of recidivism for patients when discharged into the community. 


\section{Acknowledgements}

Funding for this project was provided by the NSW Health Education and Training Institute (HETI) via the Mental Health Research Award and Workplace Learning Grant. A NSW Mental Health Commission Lived Experience Framework grant also supported the adaptations to ensure the programme suited an Australian forensic setting. This project would not have been possible without the participation of the NSW Forensic Hospital patients and allied health team whose dedication allowed the pilot to run seamlessly.

All procedures followed were in accordance with the ethical standards of the responsible committee on human experimentation (institutional and national) and with the Helsinki Declaration of 1975, as revised in 2000. Informed consent was obtained from all patients for being included in the study.

\section{Conflict of interest}

Daniel McFadden, Dr Katrina Prior and Dr Emma Barrett confirm that they have no conflict of interest to declare. 


\section{List of Tables}

Table I. Substance use treatment programme (SUTP) session plan outline

Table II. Summary of the validated scales used in the current study

Table III. Sociodemographic, mental health, substance use and substance use treatment characteristics of the sample

Table IV. Qualitative findings relating to satisfaction with SUTP

Table V. Baseline and post-intervention scores, $p$-values and effects sizes for each outcome variable

\section{List of Figures}

Figure I. Study flow diagram for the SUTP 


\section{References}

Andersson, H. W., Otterholt, E., \& Gråwe, R. W. (2017). Patient satisfaction with treatments and outcomes in residential addiction institutions. Nordic Studies on Alcohol and Drugs, 34(5), 375-384. doi:10.1177/1455072517718456

Aslan, S., Türkçapar, M. H., Eser, H. Y., \& Uğurlu, M. (2012). Reliability and validity of Beliefs about Substance Use (BSU) questionnaire in alcohol dependent patients. Bilişsel Davranışçı Psikoterapi ve Araştırmalar Derg, 1, 162-170.

Attkisson, C., \& Greenfield, T. (1996). The Client Satisfaction Questionnaire (CSQ) Scales In B. S. Dickey, L. (Ed.), Outcome Assessment in Clinical Practice (pp. 120-127). Baltimore, Maryland: Williams \& Wilkins.

Attkisson, C. C., \& Zwick, R. (1982). The client satisfaction questionnaire: Psychometric properties and correlations with service utilization and psychotherapy outcome. Evaluation and Program Planning, 5(3), 233-237.

Australian Bureau of Statistics. (2017). Australia's Population by Country of Birth. Retrieved 06/05/2020, from Australian Bureau of Statistics https://www.abs.gov.au/ausstats/abs@.nsf/Previousproducts/3412.0Main\%20Features3201516? opendocument \&tabname $=$ Summary $\&$ prodno $=3412.0 \&$ issue $=2015-16 \&$ num $=\&$ view $=$

Babor, T., Higgins-Biddle. J., Saunders, J., \& Monteiro, M. (2001). AUDIT. The Alcohol Use Disorders Identification Test: Guidelines for Use in Primary Care. Geneva, Switzerland: World Health Organisation

Barrett, E. L., Indig, D., Sunjic, S., Sannibale, C., Sindicich, N., Rosenfeld, J., . . Mills, K. (2015). Treating Comorbid Substance Use and Traumatic Stress among Male Prisoners: A Pilot Study of the Acceptability, Feasibility, and Preliminary Efficacy of Seeking Safety. International Journal of Forensic Mental Health, 14(1), 45-55. doi:10.1080/14999013.2015.1014527

Barrett, E. L., Teesson, M., \& Mills, K. L. (2014). Associations between substance use, post-traumatic stress disorder and the perpetration of violence: A longitudinal investigation. Addictive Behaviors 39(6), 1075-1080. doi:10.1016/j.addbeh.2014.03.003 
Baxter, R., Rabe-Hesketh, S., \& Parrott, J. (1999). Characteristics, needs and reoffending in a group of patients with schizophrenia formerly treated in medium security. The Journal of Forensic Psychiatry, 10(1), 69-83. doi:10.1080/09585189908402140

Beck, A. T., Wright, F. D., Newman, C. F., \& Liese, B. S. (1993). Cognitive Therapy of Substance Abuse. New York, NY: Guildford Press.

Berman, A. B., H., Palmtierna, T. \& Schlyter, F. (2003). DUDIT. The Drug Use Disorders Identification Test. Stockholm, Sweden: Karolinska Institutet, Department of Clinical Neuroscience.

Boden, M. T., \& Moos, R. (2009). Dually diagnosed patients' responses to substance use disorder treatment. Journal of Substance Abuse Treatment, 37(4), 335-345. doi:10.1016/j.jsat.2009.03.012

Boyd-Caine, T., \& Chappell, D. (2005). The forensic patient population in New South Wales. Current Issues in Criminal Justice, 17(1), 5-29.

Butler, T., Andrews, G., Allnutt, S., Sakashita, C., Smith, N. E., \& Basson, J. (2006). Mental Disorders in Australian Prisoners: A Comparison with a Community Sample. Australian \& New Zealand Journal of Psychiatry, 40(3), 272-276. doi:10.1080/j.1440-1614.2006.01785.x

Butler, T., Indig, D., Allnutt, S., \& Mamoon, H. (2011). Co-occurring mental illness and substance use disorder among Australian prisoners. Drug and Alcohol Review, 30(2), 188-194. doi:10.1111/j.1465-3362.2010.00216.x

Carlson, M. J., \& Gabriel, R. M. (2001). Patient Satisfaction, Use of Services, and One-Year Outcomes in Publicly Funded Substance Abuse Treatment. Psychiatric Services, 52(9), 12301236. doi:10.1176/appi.ps.52.9.1230

Clarke, M., Davies, S., Hollin, C., \& Duggan, C. (2011). Long-Term Suicide Risk in Forensic Psychiatric Patients. Archives of Suicide Research, 15(1), 16-28. doi:10.1080/13811118.2011.539951

Cohen, J. (2013). Statistical Power Analysis for the Behavioral Sciences. Burlington, Canada: Elsevier Science. 
De Burca, C., Miles, H., \& Vasquez, E. (2013). Substance use amongst mentally disordered offenders in medium security: prevalence and relationship to offending behaviour. Journal of Forensic Practice, 15(4), 259-268. doi:10.1108/JFP-08-2012-0010

Dekker, J. (2010). An evaluation of the compulsory drug treatment program (CDTP). Sydney, Australia: NSW Bureau of Crime Statistics and Research.

Derry, A., \& Batson, A. (2008). Getting out and staying out: does substance use treatment have an effect on outcome of mentally disordered offenders after discharge from medium secure service? British Journal of Forensic Practice, 10(2), 13-17. doi:10.1108/14636646200800009

Dolan, M., \& Kirwan, H. (2001). Survey of staff perceptions of illicit drug use among patients in a medium secure unit. Psychiatric Bulletin, 25(1), 14-17. doi:10.1192/pb.25.1.14

Donnelly, V., Lynch, A., Devlin, C., Naughten, L., Gibbons, O., Mohan, D., \& Kennedy, H. G. (2011). Therapeutic alliance in forensic mental health: coercion, consent and recovery. Irish Journal of Psychological Medicine, 28(1), 21-28. doi:10.1017/S0790966700011861

Eagle, K., Ma, T., \& Sinclair, B. (2019). Integrated substance use rehabilitation in a secure forensic facility. Journal of Forensic Practice, 21(1), 50-60. doi:10.1108/JFP-09-2018-0037

Ersche, K. D., Turton, A. J., Croudace, T., \& Stochl, J. (2012). Who Do You Think Is in Control in Addiction? A Pilot Study on Drug-related Locus of Control Beliefs. Addictive Disorders \& Their Treatment, 11(4), 173-223. doi:10.1097/ADT.0b013e31823da151

Fazel, S. (2012). Severe mental illness in 33588 prisoners worldwide: systematicreview and metaregression analysis. The British Journal of Psychiatry, 200(5), 364-373. doi:10.1192/bjp.bp.111.096370

Fazel, S., Bains, P., \& Doll, H. (2006). Substance abuse and dependence in prisoners: a systematic review. 101(2), 181-191. doi:10.1111/j.1360-0443.2006.01316.x

Fritz, C. O., Morris, P. E., \& Richler, J. J. (2012). Effect size estimates: current use, calculations, and interpretation. Journal of Experimental Psychology: General, 141(1), 2. doi:10.1037/a0024338

Hall, E. (2001). Feelings About Drug Use Drug-Related Locus of Control. California, USA: UCLA Integrated Substance Abuse Programs. 
Hayes, H., Kemp, R. I., Large, M. M., \& Nielssen, O. B. (2014). A 21-year retrospective outcome study of New South Wales forensic patients granted conditional and unconditional release. Australian \& New Zealand Journal of Psychiatry, 48(3), 259-282. doi: $10.1177 / 0004867413507610$

Horvath, A., \& Symonds, B. (1991). Relation Between Working Alliance and Outcome in Psychotherapy: A Meta-Analysis. Journal of Counseling Psychology, 38(2), 139. doi:10.1037/0022-0167.38.2.139

Horvath, A. \& Symonds, D. (1991). Relation Between Working Alliance and Outcome in Psychotherapy: A Meta-Analysis. Journal of Counseling Psychology, 38(2), 139-149. doi:10.1037/0022-0167.38.2.139

Jhanjee, S. (2014). Evidence based psychosocial interventions in substance use. Indian Journal of Psychological Medicine, 36(2), 112-119. doi:10.4103/0253-7176.130960

Johnson, C., Smith, J., Crowe, C., \& Donovan, M. (1993). Suicide among Forensic Psychiatric Patients. Medicine, Science and the Law, 33(2), 137-143. doi:10.1177/002580249303300209 Justice Health and Forensic Mental Health Network (JHFMHN). (2016). Forensic Mental Health Patient Survey Report. Sydney, Australia: Justice Health and Forensic Mental Health Network.

Kelly, P. J., Kyngdon, F., Ingram, I., Deane, F. P., Baker, A. L., \& Osborne, B. A. (2018). The Client Satisfaction Questionnaire-8: Psychometric properties in a cross-sectional survey of people attending residential substance abuse treatment. Drug and Alcohol Review, 37(1), 79-86. doi:10.1111/dar.12522

Kourmousi, N., Xythali, V., \& Koutras, V. (2015). Reliability and Validity of the Multidimensional Locus of Control IPC Scale in a Sample of 3668 Greek Educators. Social Sciences, 4(4), 1067-1078. doi:10.3390/socsci4041067

Landenberger, N. A., \& Lipsey, M. W. (2005). The positive effects of cognitive-behavioral programs for offenders: A meta-analysis of factors associated with effective treatment. Journal of Experimental Criminology, 1(4), 451-476. doi:10.1007/s11292-005-3541-7 
Levenson, H. (1973). Multidimensional Locus of Control in Psychiatric Patients. Journal of Consulting and Clinical Psychology, 41(3), 397. doi:10.1037/h0035357

Lipsey, M. W., Chapman, G. L., \& Landenberger, N. A. (2001). Cognitive-Behavioral Programs for Offenders. The Annals of the American Academy of Political and Social Science, 578, 144157.

Loewenthal, K. (2018). An Introduction to Psychological Tests and Scales (Second edition). Boca Raton, Florida: Psychology Press.

Long, C. G., Fulton, B., Fitzgerald, K.-A., \& Hollin, C. R. (2010). Group substance abuse treatment for women in secure services. Mental Health and Substance Use, 3(3), 227-237. doi:10.1080/17523281.2010.506182

McHugh, R. K., Hearon, B. A., \& Otto, M. W. (2010). Cognitive behavioral therapy for substance use disorders. The Psychiatric clinics of North America, 33(3), 511-525. doi:10.1016/j.psc.2010.04.012

McMurran, M. (2002). Expert Paper: Dual Diagnosis of Mental Disorder and Substance Misuse. London, United Kingdom: Department of Health

Miles, H. (2015). “A new horizon?”: evaluation of an integrated Substance Use Treatment Programme (SUTP) for mentally disordered offenders. Advances in Dual Diagnosis, 8(2), 90101. doi:10.1108/ADD-03-2015-0002

Miles, H., Dutheil, L., Welsby, I., \& Haider, D. (2007). 'Just Say No': A preliminary evaluation of a three-stage model of integrated treatment for substance use problems in conditions of medium security. Journal of Forensic Psychiatry \& Psychology, 18(2), 141-159. doi:10.1080/14789940601101897

Morris, C., \& Moore, E. (2009). An evaluation of group work as an intervention to reduce the impact of substance misuse for offender patients in a high security hospital. The Journal of Forensic Psychiatry \& Psychology, 20(4), 559-576. doi:10.1080/14789940802263736

Mpofu, E., Athanasou, J. A., Rafe, C., \& Belshaw, S. H. (2018). Cognitive-Behavioral Therapy Efficacy for Reducing Recidivism Rates of Moderate- and High-Risk Sexual Offenders: A 
Scoping Systematic Literature Review. International Journal of Offender Therapy and Comparative Criminology, 62(1), 170-186. doi:10.1177/0306624X16644501

National Institute of Health and Care Excellence. (2007). Drug misuse in over 16s: Psychosocial interventions (NICE Clinical Guideline No. 51). London, United Kingdom: National Institute of Health and Care Excellence. Retrieved from https://www.nice.org.uk/guidance/cg51/chapter/Introduction

Oddie, S., \& Davies, J. (2009). A Multi-Method Evaluation of a Substance Misuse Program in a Medium Secure Forensic Mental Health Unit. Journal of Addictions Nursing, 20(3), 132-141. doi:10.1080/10884600903078944

Ogloff, J. R., Lemphers, A., \& Dwyer, C. (2004). Dual diagnosis in an Australian forensic psychiatric hospital: prevalence and implications for services. Behavioral Sciences \& the Law 22(4), 543562. doi:10.1002/bsl.604

Paap, D., Schrier, E., \& Dijkstra, P. U. (2019). Development and validation of the Working Alliance Inventory Dutch version for use in rehabilitation setting. Physiotherapy Theory and Practice, 35(12), 1292-1303. doi:10.1080/09593985.2018.1471112

Pickard, H., \& Fazel, S. (2013). Substance abuse as a risk factor for violence in mental illness: some implications for forensic psychiatric practice and clinical ethics. Current Opinion in Psychiatry 26(4), 349-354. doi:10.1097/YCO.0b013e328361e798

Ritchie, G., Weldon, S., Freeman, L., MacPherson, G., \& Davies, K. (2011). Outcomes of a drug and alcohol relapse prevention programme in a population of mentally disordered offenders. British Journal of Forensic Practice, 13(1), 32-43.

Sandbrook, J., Clark, T., \& Cocksedge, K. A. (2015). Addressing substance misuse in medium secure settings in the UK and Ireland - a survey of current practice. The Journal of Forensic Practice, 17(3), 192-203. doi:10.1108/JFP-01-2015-0006

Scott, F., Whyte, S., Burnett, R., Hawley, C., \& Maden, T. (2004). A national survey of substance misuse and treatment outcome in psychiatric patients in medium security. The Journal of Forensic Psychiatry \& Psychology, 15(4), 595-605. doi:10.1080/14789940412331305556 
Shepherd, S. M. (2017). Aboriginal prisoners with cognitive impairment:is this the highest risk group? Trends and Issues in Crime and Criminal Justice. (536), 1-14.

Sklar, S. M., Annis, H. M., \& Turner, N. E. (1997). Development and validation of the Drug-Taking Confidence Questionnaire: A measure of coping self-efficacy. Addictive Behaviors, 22(5), 655-670. doi:10.1016/S0306-4603(97)00006-3

Sklar, S. M., \& Turner, N. E. (1999). A brief measure for the assessment of coping self-efficacy among alcohol and other drug users. Addiction, 94(5), 723-729.

Smith, N., \& Trimboli, L. (2010). Comorbid substance and non-substance mental health disorders and re-offending among NSW prisoners (Report No. 10301046). Crime and Justice Bulletin.

Sturgiss, E. A., Rieger, E., Haesler, E., Ridd, M. J., Douglas, K., \& Galvin, S. L. (2019). Adaption and validation of the Working Alliance Inventory for General Practice: qualitative review and cross-sectional surveys. Family Practice, 36(4), 516. doi:10.1093/fampra/cmy113

Tibber, M. S., Piek, N., \& Boulter, S. (2015). Preliminary evaluation of a forensic dual diagnosis intervention. Advances in Dual Diagnosis, 8(1), 42-56. doi:10.1108/ADD-08-2014-0019

Tyler, N., Miles, H. L., Karadag, B., \& Rogers, G. (2019). An updated picture of the mental health needs of male and female prisoners in the UK: prevalence, comorbidity, and gender differences.(Report). Social Psychiatry and Psychiatric Epidemiology, 54(9), 1143. doi:10.1007/s00127-019-01690-1

Urbanoski, K. A., \& Wild, T. C. (2012). Assessing self-determined motivation for addiction treatment: validity of the Treatment Entry Questionnaire. Journal of Substance Abuse Treatment, 43(1), 70-79. doi:10.1016/j.jsat.2011.10.025

Wood, N., Patel, K., Skinner, J., \& Thomson, K. (2009). Setting up a dual diagnosis service within a forensic inpatient setting: reflections one year on. Advances in Dual Diagnosis, 2(3), 20-24. doi:10.1108/17570972200900021

Wright, N., Walters, P., \& Strang, J. (2016). Dual diagnosis in prisons: management of co-existing substance use and mental health disorders. Advances in Dual Diagnosis, 9(1),. doi:10.1108/ADD-12-2015-0025 